\section{Ce qu'il faut savoir sur le TarMed}

\author{
Groupe de travail TarMed de la FMH
}

$d r$. TarMed, voilà un mot bien connu des milieux intéressés dans le domaine de la santé, mais que recouvre concrètement ce terme, quelles sont les parties concernées et comment se déroulent les négociations. Ce sont là des questions auxquelles le résumé ci-après se propose de répondre.

\section{Parties à la convention TarMed}

Dans le cadre du projet TarMed, les parties à la convention sont:

- le Concordat des assureurs-maladie suisses (CAMS);

- la Commission des tarifs médicaux LAA (CTM), l'Assurance militaire (AM), l'Assurance-invalidité (AI);

- la Fédération des médecins suisses (FMH);

- H+ Les Hôpitaux de Suisse.

\section{Parties à la convention TarMed en détail}

\section{Répondants des frais (assureurs)}

- CAMS: Organisation faîtière des assureurs-maladie de Suisse et, de ce fait, représentante du domaine LAMal.

- CTM, AM, AI: Les assureurs "fédéraux"; autrement dit, les assureurs (de l'Etat ou de droit public) qui œuvrent dans le domaine AA, AM et AI.

Fournisseurs de prestations

- FMH: Organisation faîtière des médecins.

- H+: Organisation faitière des hôpitaux de Suisse.

Statut d'observateur

- Conférence des directeurs cantonaux des affaires sanitaires (CDS).

\section{Organes du TarMed}

Direction générale du projet TarMed

Celle-ci comprend les hauts responsables des parties à la convention, à savoir: le Dr P. Saladin $(\mathrm{H}+)$, le $\mathrm{Dr}$ W. Morger (CTM), M. M. Manser (CAMS) et le Dr. H. H. Brunner (FMH). Cet organe peut être comparé à un conseil d'administration qui assume en dernier lieu la responsabilité pour l'ensemble du projet.
Direction du projet TarMed

Elle comprend les délégués des parties à la convention, désignés pour prendre part aux négociations. La CDS y a un statut d'observateur.

\section{Secrétariat TarMed}

Est tenu par le Service central des tarifs médicaux LAA (SCTM) de la CNA.

\section{TarMed et accords bilatéraux}

Tant dans le domaine LAA que LAMal, des accords bilatéraux sont conclus entre les répondants des frais (assureurs) et les fournisseurs de prestations (médecins, hôpitaux).

\section{Domaine LAA}

\section{Assureurs - corps médical}

(autrement dit: $C T M / A M / A I-F M H$ )

Les assureurs "fédéraux" et le corps médical ont conclu la convention TarMed conformément aux dispositions de la LAA, de la LAI, de la LAM et des ordonnances s'y rapportant. Ladite convention porte sur les prestations fournies dans le cadre du TarMed par les médecins exerçant à titre indépendant.

\section{Assureurs - hôpitaux}

(autrement dit: $C T M / A M / A I-H+$ )

A l'instar de la convention entre les assureurs et les médecins, les assureurs et $\mathrm{H}+$ passent également une convention. Celle-ci règle les dispositions concernant les prestations fournies par les médecins salariés dans le cadre du TarMed.

\section{Domaine LAMal}

Assureurs - corps médical

(autrement dit: CAMS - FMH)

Etant donné que l'application de la LAMal est soumise à la souveraineté des cantons, le CAMS et la FMH peuvent uniquement conclure une convention sur le plan fédéral. Ladite convention fixe le cadre pour les négociations sur le plan cantonal. En d'autres termes, les conventions cantonales entres les fédérations cantonales de caisses-maladie et les sociétés cantonales de médecine concernant les prestations fournies par les médecins exerçant à titre indépendant doivent satisfaire aux exigences de la convention-cadre.

Assureurs - hôpitaux (autrement dit: CAMS - H+) A l'instar de la convention entre le CAMS et la FMH, une convention est passée entre le CAMS et H+. Celleci fixe les dispositions concernant les prestations fournies dans le cadre du TarMed par les médecins salariés. 


\section{Nouvelle organisation TarMed (NOT)}

L'introduction du TarMed dans les domaines LAA et LAMal met fin au projet. L'entretien de la structure tarifaire devra cependant être garanti. Pour ce faire, une nouvelle organisation TarMed sera créée, à laquelle prendront également part les délégués des parties à la convention.

\section{Délégués TarMed de la FMH}

Les personnes et organes énumérés ci-après sont chargés de régler les différentes tâches se rapportant au TarMed:

- Comité central de la FMH: haute surveillance;

- Président de la FMH: responsable de la délégation aux négociations LAA; membre de la délégation aux négociations LAMal, membre de la direction générale du projet;

- Président du Comité des sociétés cantonales de médecine (G7): il est principalement chargé de mener les négociations dans le domaine LAMal.

- Responsable du service tarifaire: responsable du projet TarMed, membre de la Commission tarifaire (CoTa);

- CoTa: Commission tarifaire de la FMH; poste stratégique du responsable du projet TarMed. 